\title{
Highlights of Planck results
}

\author{
Jan Tauber*广 \\ European Space Agency \\ Scentific Support Office \\ Keplerlaan 1 \\ 2201AZ Noordwijk \\ The Netherlands \\ E-mail: jtauber@cosmos.esa.int
}

The aim of my presentation was to summarize the main cosmological results obtained so far from the Planck mission, and to point to the wide variety of data products available from the Planck Legacy Archive. I highlighted the concordance of Planck's cosmological model with other independent probes, and noted some of the tensions. A new and higher-quality set of data products based on Planck will be released to the community in late 2017. This paper summarizes the presentation and includes the main references to be looked up by anyone interested in Planck.

XII Multifrequency Behaviour of High Energy Cosmic Sources Workshop

\section{2-17 June}

Palermo, Italy

\footnotetext{
* Speaker.

${ }^{\dagger}$ On behalf of the Planck Collaboration.
} 
The Planck satellite (https://www.cosmos.esa.int/web/Planck) was launched on 14 May 2009 and observed the sky between 12 August 2009 and 23 October 2013. Planck's scientific payload contained an array of 74 detectors in nine bands covering frequencies between 25 $\mathrm{GHz}$ and $1000 \mathrm{GHz}$, which scanned the sky with angular resolution between 33 and 5 arcminutes. The main objective of Planck, defined in 1995, was to measure the spatial anisotropies in the temperature of the cosmic microwave background (CMB). The first major set of scientific data from Planck, consisting mainly of temperature maps of the whole sky produced from the first 15 months of acquired data, was released to the public in March 2013. These data and associated scientific results were described in a special issue of Astronomy and Astrophysics (vol. 571, 2014); an overview of these results can be found in Planck Collaboration I (2014).

Between February and July 2015, all of the data acquired by Planck were released to the public. Processed products included temperature and polarisation all-sky maps (Figs. 1 and 2). This data release was accompanied by 28 papers authored by the Planck Collaboration, describing the reduction of the data and the major scientific results. One of these papers (Planck Collaboration I 2016) contains a detailed overview of products and results and is a recommended introduction to Planck. All of the papers by the Planck Collaboration can be found via https://www . cosmos.esa.int/web/Planck, and all of the data products are accessible online via the Planck Legacy Archive, https://www.cosmos.esa.int/web/planck/pla. Continuing efforts to improve the quality of the data will lead to a third and final release of Planck products in late 2017, which will represent the legacy of the mission.
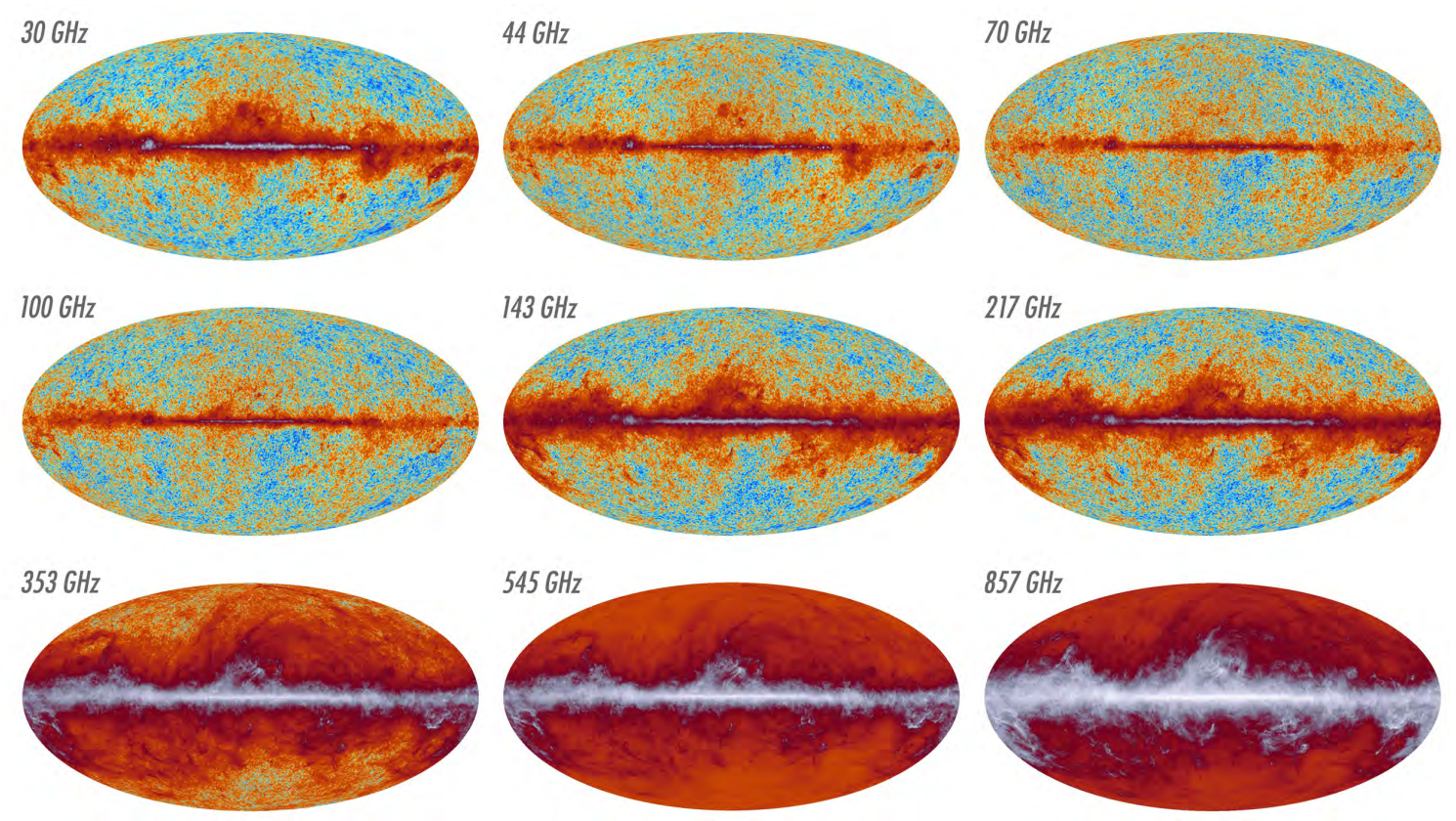

Figure 1: The nine temperature maps from 30 to $857 \mathrm{GHz}$. The colour scale is tailored to show the full dynamic range of the maps. (credit: ESA and the Planck Collaboration).

The 2015 Planck CMB temperature maps have significantly lower noise than those produced in 2013. Between 2013 and 2015 the understanding of Planck beams, pointing, calibration and 

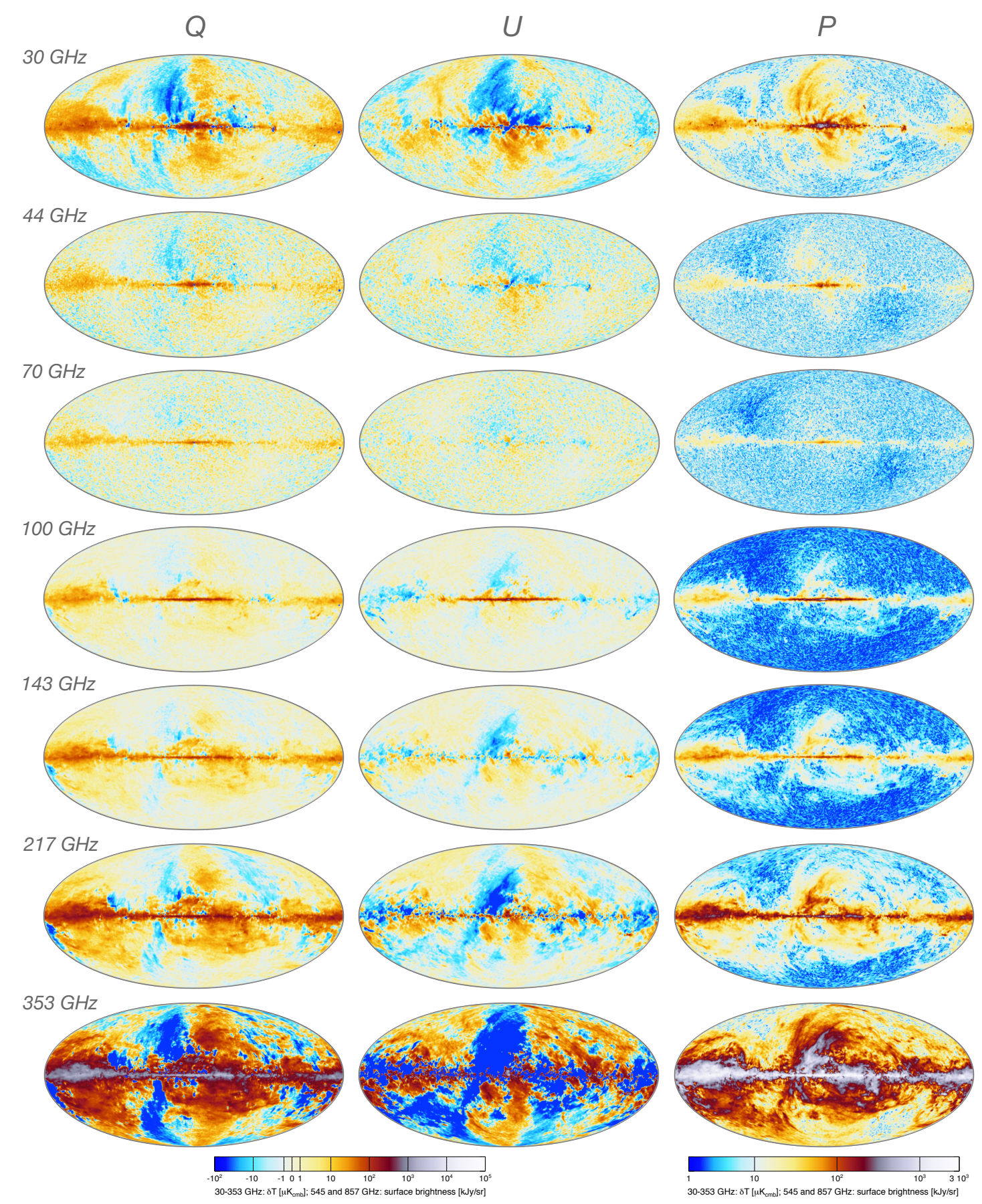

Figure 2: The seven Planck polarisation maps from $30 \mathrm{GHz}$ to $353 \mathrm{GHz}$, shown in Stokes Q and U, and in total polarised intensity $(\mathrm{P})$. The colour scale is tailored to show the full dynamic range of the maps. (credit: ESA and the Planck Collaboration). 
systematic errors has significantly advanced. All of these factors have led to improvements in the cosmological results of Planck. The all-sky polarisation maps between $30 \mathrm{GHz}$ and $353 \mathrm{GHz}$ (see Fig. 2) are a major new release of 2015 and provide an entirely new view of the sky. They allow the extraction of maps of the polarised CMB anisotropies, which not only carry new cosmological information, but also provide a unique probe of the thermal history of the Universe during the time when the first stars and galaxies formed. In addition, the polarisation data can be used to estimate cosmological parameters independently of temperature, in effect constituting important confirmation of the Planck temperature-based results.

The best-fit 2015 cosmological parameters (Table 1, from Planck Collaboration XIII (2016)) confirm the basic 6-parameter Lambda cold dark matter scenario that was first established in 2013, with reduced uncertainties. There is no compelling evidence for any extensions to the 6-parameter model, or any need for new physics. Depending somewhat on the precise data combinations used, five of the six parameters have now been measured to better than $1 \%$ precision. At large angular scales, it is now possible to use Planck-only products to carry out polarisation-based cosmological analysis. Specifically, the optical depth of re-ionisation, $\tau$, can be estimated independently of other experiments. A preview of the improved quality of the forthcoming Legacy polarisation data can be appreciated in Planck Collaboration Int. XLVI (2016) and Planck Collaboration Int. XLVII (2016). The value of $\tau$ found in these papers $(0.055 \pm 0.009)$ is lower and more precise than in previous determinations (still consistent with the 2015 estimate), implying later and faster reionisation, which resolves important open questions related to the formation of the first ionizing objects (Planck Collaboration Int. XLVII 2016).

While the Planck $\Lambda$ CDM model is in good agreement with many other cosmological probes (Planck Collaboration I 2016), some mild tensions exist. One of these, which has received a good deal of attention in the literature, relates to the Hubble constant $\mathrm{H}_{0}$. Indeed, the value of $\mathrm{H}_{0}$ derived from Planck CMB analysis is in tension with the most precise local-distance-ladder determination (Riess et al. 2016) at the level of $\sim 3 \sigma$. The dichotomy between totally independent estimates points either to new physics or to unaccounted systematic effects in one of the two measurements (Freedman 2017). Addison et al. (2016) noted that limiting the Planck CMB data only to the largest angular scales (those accessible to WMAP) brought the estimate of $\mathrm{H}_{0}$ into consistency with the local value, and suggested that the current tension could be due to systematic effects in the Planck data at small angular scales. However, Planck Collaboration LI (2016) showed that the effect noted by Addison et al. (2016) can be largely attributed to the well-known "low- $\ell$ anomaly" or deficit of power in the $\mathrm{CMB}$ temperature angular power spectrum at $\ell \sim 10-30$, which biasses significantly the determination of cosmological parameters based on measurements limited to $\ell<1000$. In effect, it is required to measure a very wide range of angular scales (only accessible to Planck so far) in order to counteract this bias. A more recent paper by Addison et al. (2017) confirms that the tension between the Planck CMB analysis and the distance-ladder estimate cannot be due to systematic effects in the Planck data at high- $\ell$ s. It therefore remains a distinct possibility that the local value of $\mathrm{H}_{0}$ is influenced by additional physics (Riess et al. 2016) or possibly by local effects e.g. our situation within a void (Hoscheit \& Barger 2017; Keenan et al. 2016).

In addition to their value for cosmology, the Planck data are an important resource for astrophysics. The 2015 data allow the production of high-quality maps of synchrotron, free-free, spinning dust, thermal dust and carbon monoxide emissions from the Milky Way; and for the first 
time, polarised synchrotron and dust emission maps of our Galaxy are available for detailed investigation (Planck Collaboration X 2016; Planck Collaboration XXV 2016). In addition, catalogues of galactic and extragalactic compact sources have been produced (Planck Collaboration XXVI 2016; Planck Collaboration XXVII 2016; Planck Collaboration XXVIII 2016), allowing a wide variety of astrophysical studies.

Table 1: Column 2 shows parameter best-fit values and $68 \%$ confidence levels for the base $\Lambda$ CDM cosmology, computed from the 2015 Planck CMB temperature ("TT") and low-ell ("lowP") polarisation power spectra, in combination with the Planck CMB lensing likelihood ("lensing"). In the third column, the addition of CMB high- $\ell$ polarisation data and a compilation of external data sets ("ext") leads to reduced uncertainties. While we see no evidence that systematics in the high $\ell$ polarisation are biasing parameters in the base $\Lambda$ CDM model, a conservative choice would be to take the parameters listed in column 2 . The first 6 rows contain the base $\Lambda \mathrm{CDM}$ model parameters, all others being derived from them. For more details see Planck Collaboration I (2016) and Planck Collaboration XIII (2016).

\begin{tabular}{|c|c|c|}
\hline Parameter & PlanckTT+lowP+lensing & $\begin{array}{l}\text { PlanckTT,TE,EE+ } \\
\text { lowP+lensing+ext }\end{array}$ \\
\hline$\Omega_{\mathrm{b}} h^{2}$. & $0.02226 \pm 0.00023$ & $0.02230 \pm 0.00014$ \\
\hline$\Omega_{\mathrm{c}} h^{2}$. & $0.1186 \pm 0.0020$ & $0.1188 \pm 0.0010$ \\
\hline $100 \theta_{\mathrm{MC}} \ldots$ & $1.04103 \pm 0.00046$ & $1.04093 \pm 0.00030$ \\
\hline$\tau \ldots \ldots$ & $0.066 \pm 0.016$ & $0.066 \pm 0.012$ \\
\hline $\ln \left(10^{10} A_{\mathrm{s}}\right)$ & $3.062 \pm 0.029$ & $3.064 \pm 0.023$ \\
\hline$n_{\mathrm{s}} \ldots \ldots \ldots$ & $0.9677 \pm 0.0060$ & $0.9667 \pm 0.0040$ \\
\hline$H_{0} \ldots$ & $67.8 \pm 0.9$ & $67.74 \pm 0.46$ \\
\hline$\Omega_{\Lambda} \ldots \ldots$ & $0.692 \pm 0.012$ & $0.6911 \pm 0.0062$ \\
\hline$\Omega_{\mathrm{b}} \ldots \ldots$ & $0.0484 \pm 0.0010$ & $0.04860 \pm 0.00051$ \\
\hline$\Omega_{\mathrm{c}} \ldots \ldots$ & $0.258 \pm 0.011$ & $0.2589 \pm 0.0057$ \\
\hline$\Omega_{\mathrm{m}} \ldots \ldots$ & $0.308 \pm 0.012$ & $0.3089 \pm 0.0062$ \\
\hline$\Omega_{\mathrm{m}} h^{2} \ldots \ldots$ & $0.1415 \pm 0.0019$ & $0.14170 \pm 0.00097$ \\
\hline$\Omega_{\mathrm{m}} h^{3} \ldots$ & $0.09591 \pm 0.00045$ & $0.09598 \pm 0.00029$ \\
\hline$\sigma_{8} \ldots \ldots$ & $0.815 \pm 0.009$ & $0.8159 \pm 0.0086$ \\
\hline$\sigma_{8} \Omega_{\mathrm{m}}^{0.5} \ldots$ & $0.4521 \pm 0.0088$ & $0.4535 \pm 0.0059$ \\
\hline $\operatorname{Age}[\mathrm{Gyr}] \ldots$ & $13.799 \pm 0.038$ & $13.799 \pm 0.021$ \\
\hline$r_{\text {drag }} \ldots \ldots$ & $147.60 \pm 0.43$ & $147.50 \pm 0.24$ \\
\hline$k_{\text {eq }} \ldots \ldots$ & $0.01027 \pm 0.00014$ & $0.010288 \pm 0.000071$ \\
\hline
\end{tabular}

Acknowledgements The development of Planck has been supported by: ESA; CNES and CNRS/INSUIN2P3-INP (France); ASI, CNR, and INAF (Italy); NASA and DoE (USA); STFC and UKSA (UK); CSIC, MICINN, JA and RES (Spain); Tekes, AoF and CSC (Finland); DLR and MPG (Germany); CSA (Canada); DTU Space (Denmark); SER/SSO (Switzerland); RCN (Norway); SFI (Ireland); FCT/MCTES (Portugal); and PRACE (EU). A description of the Planck Collaboration and a list of its members, including the technical or scientific activities in which they have 
been involved, can be found at https://www.sciops.esa.int/index.php?project= planck\&page $=$ Planck_Collaboration.

\section{References}

Addison, G. E., Huang, Y., Watts, D. J., et al. 2016, ApJ, 818, 132

Addison, G. E., Watts, D. J., Bennett, C. L., et al. 2017, ArXiv e-prints

Freedman, W. L. 2017, Nature Astronomy, 1, 0169

Hoscheit, B. L. \& Barger, A. J. 2017, in American Astronomical Society Meeting Abstracts, Vol. 230, American Astronomical Society Meeting Abstracts, 314.05

Keenan, R. C., Barger, A. J., \& Cowie, L. L. 2016, in IAU Symposium, Vol. 308, The Zeldovich Universe: Genesis and Growth of the Cosmic Web, ed. R. van de Weygaert, S. Shandarin, E. Saar, \& J. Einasto, 295-298

Planck Collaboration I. 2014, A\&A, 571, A1

Planck Collaboration I. 2016, A\&A, 594, A1

Planck Collaboration X. 2016, A\&A, 594, A10

Planck Collaboration XIII. 2016, A\&A, 594, A13

Planck Collaboration XXV. 2016, A\&A, 594, A25

Planck Collaboration XXVI. 2016, A\&A, 594, A26

Planck Collaboration XXVII. 2016, A\&A, 594, A27

Planck Collaboration XXVIII. 2016, A\&A, 594, A28

Planck Collaboration Int. XLVI. 2016, A\&A, 596, A107

Planck Collaboration Int. XLVII. 2016, A\&A, 596, A108

Planck Collaboration LI. 2016, A\&A, submitted

Riess, A. G., Macri, L. M., Hoffmann, S. L., et al. 2016, ApJ, 826, 56 